\title{
Effects of Mito-TEMPO on the survival of vitrified bovine blastocysts in vitro
}

\author{
Jae-Hoon Jeong ${ }^{1,2}$, Seul-Gi Yang ${ }^{1,2}$, Hyo-Jin Park ${ }^{1,2}$ and Deog-Bon Koo ${ }^{1,2, *}$ \\ ${ }^{1}$ Department of Biotechnology, College of Engineering, Daegu University, Gyeongsan 38453, Korea \\ ${ }^{2}$ Institute of Infertility, Daegu University, Gyeongsan 38453, Korea
}

Received November 23, 2021

Revised November 30, 2021

Accepted November 30, 2021

\section{*Correspondence \\ Deog-Bon Koo \\ E-mail: dbkoo@daegu.ac.kr}

\section{Author's Position and Orcid no.}

Jeong JH, MS student,

https://orcid.org/0000-0002-0202-4765

Yang SG, PhD,

https://orcid.org/0000-0003-3247-2443

Park HJ, PhD,

https://orcid.org/0000-0003-3287-2914

Koo DB, Professor,

https://orcid.org/0000-0001-7825-9598

\begin{abstract}
Vitrification methods are commonly used for mammalian reproduction through the long-term storage of blastocyst produced in vitro. However, the survival and quality of embryos following vitrification are significantly low compared with blastocyst from in vitro production (IVP). This study evaluates that the survival of frozen-thawed bovine embryos was relevant to mitochondrial superoxide derived mitochondrial activity. Here we present supplementation of the cryopreservation medium with MitoTEMPO $(0.1 \mu \mathrm{M})$ induced a significant $(p<0.001$; non-treated group: $56.8 \pm 8.7 \%$, reexpanded at $24 \mathrm{~h}$ vs Mito-TEMPO treated group: $77.5 \pm 8.9 \%$, re-expanded at $24 \mathrm{~h}$ ) improvement in survival rate of cryopreserved-thawed bovine blastocyst. To confirm the quality of vitrified blastocyst after thawing, DNA fragmentation of survived embryos was examined by TUNEL assay. As a result, TUNEL positive cells rates of frozenthawed embryos were lower in the Mito-TEMPO treated group (4.2 $\pm 1.4 \%)$ than the non-treated group $(7.1 \pm 3.5 \%)$. In addition, we investigated the intracellular ROS and mitochondrial specific superoxide production using DCF-DA and Mito-SOX staining in survived bovine embryos following vitrification depending on Mito-TEMPO treatment. As expected, intracellular ROS levels and superoxide production of vitrified blastocysts after cryopreservation were significantly reduced $(p<0.05)$ according to Mito-TEMPO supplement in freezing medium. Also, mitochondrial activity measured by MitoTracker Orange staining increased in the frozen-thawed embryos with Mito-TEMPO compared with non-treated group. These results indicate that the treatment of Mito-TEMPO during cryopreservation might induce reduction in DNA fragmentation and apoptosis-related ROS production, consequently increasing mitochondrial activation for developmental capacity of frozen-thawed embryos.
\end{abstract}

Keywords: bovine blastocyst, cryopreservation, Mito-TEMPO, superoxide, vitrification medium

\section{INTRODUCTION}

Cryopreservation as a technique for reproduction is used to preserve the genetic complements on domestic animals and humans (Lee et al., 2014; Kim et al., 2020). Especially, cryopreservation technology provides ad- ditional opportunity of pregnancy through the vitrifiedwarmed process in animal embryos. This is applied as a necessary technique for pregnancy using in vitro fertilization (IVF) embryos to solve the difficulty of female infertility (Hara et al., 2018). However, cryopreservation method is accompanied by cryoinjury such as the reactive 
oxygen species (ROS) production (Len et al., 2019), DNA fragmentation (Inaba et al., 2016), mitochondria dysfunctions (Gualtieri et al., 2021), and physiological damage from small ice crystals (Huebinger et al., 2016). Therefore, development of new cryopreservation additives is necessary to protect from the ROS production, mitochondrial related cryoinjury, and apoptosis during cryopreservedthawed process.

Vitrified embryos following freezing are known to increase mitochondrial dysfunctions such as loss of mitochondrial membrane potential (MMP) and adenosine triphosphate (ATP) productions compared with fresh embryos in mammals (Nohales-Córcoles et al., 2016). Mitochondria are important intracellular organelle for cell survival in mammalian cells and embryos (Babayev and Seli, 2015; Xia et al., 2019). Mitochondria play a critical role in producing ATP through the electron transport chain, which assists oocyte maturation and embryo development competence in mammalian embryos (May-Panloup et al., 2021). In addition, the ATP synthesis process accompanies ROS which is a direct by-product from mitochondria (van Hameren et al., 2019). In general, mitochondria-derived superoxide is known to carry out the role of redox-sensitive second messenger for generating signal responses in multiple ways (Shadel and Horvath, 2015). Whereas, excessive oxidative stress by external damage such as freezing and thawing process impair cellular functions and lead to apoptosis (Ott et al., 2007; Lee et al., 2016; Len et al., 2019).

Triphenylphosphonium chloride (Mito-TEMPO) is a potent cell-permeable ROS antioxidant, which is known as a mitochondrial-derived superoxide target scavenger (Yang et al., 2019). Also, Mito-TEMPO is a synthetic compound that can play a role as superoxide dismutase (SOD) $\mathrm{mi}^{-}$ metic function in mammalian cells and embryos (Asadzadeh et al., 2021). Mito-TEMPO has an antioxidant capacity that protects cells against oxidative damages in human pathologies (Dikalova et al., 2010; Choumar et al., 2011). According to previous study, reduction of superoxidederived oxidative stress by Mito-TEMPO improved oocyte maturation rate and developmental competence in porcine and bovine embryos (Yang et al., 2018; Park et al., 2020; Yousefian et al., 2021). Moreover, freezing medium with Mito-TEMPO supplementation assisted conservation of post-thawed sperm quality and survival competence in human (Lu et al., 2018; Masoudi et al., 2021). However, studies concerning the protective effects of mitochondrial specific superoxide production by Mito-TEMPO on bovine embryos and blastocyst during cryopreservation have not been reported.

Therefore, we hypothesized that Mito-TEMPO could protect the low survival of cryopreserved-thawed bovine blastocysts in response to damages from the ROS or superoxide production. In the present study, we confirmed the effects of Mito-TEMPO supplementation during the cryopreservation process on survival, intracellular ROS levels, superoxide production and mitochondrial activity in vitrified-warmed bovine embryos.

\section{MATERIALS AND METHODS}

\section{Chemicals}

Unless otherwise stated, all chemicals used in this study were purchased from Sigma Chemical Co. (St. Louis, MO, USA).

\section{In vitro production of bovine embryos}

Bovine ovaries were acquired from a local abattoir and transported to the laboratory in $0.9 \%$ saline containing 75 $\mu \mathrm{g} / \mathrm{mL}$ penicillin $\mathrm{G}$ sodium salt at $36-38^{\circ} \mathrm{C}$. Cumulus-oocyte complexes (COCs) were aspirated from follicles (3-6 $\mathrm{mm}$ in diameter) using a $10 \mathrm{~mL}$ disposable syringe with an 18-gauge needle. Approximately 15 COCs were matured in $50 \mu \mathrm{L}$ of IVM medium in a $60 \mathrm{~mm}$ dish under paraffin oil for $22 \mathrm{~h}$ at $38.5^{\circ} \mathrm{C}$ under $5 \% \mathrm{CO}_{2}$. The medium used for oocyte maturation was TCM-199 (Gibco-BRL, Grand Island, NY, USA) supplemented with $0.2 \mathrm{mM} \mathrm{Na-pyruvate,}$ $0.6 \mathrm{mM}$ cysteine, $10 \mathrm{IU} / \mathrm{mL}$ pregnant mare's serum gonadotropin (PMSG), $10 \mathrm{IU} / \mathrm{mL}$ human chorionic gonadotropin (hCG), $10 \mathrm{ng} / \mathrm{mL}$ epidermal growth factor (EGF), $25 \mu \mathrm{g} / \mathrm{mL}$ gentamycin, $25 \mu \mathrm{M} \beta$-mercaptoethanol, 10\% FBS (GibcoBRL), and $1 \mu \mathrm{g} / \mathrm{mL}$ estradiol- $\beta$. Following IVM, 15 oocytes were fertilized with frozen-thawed sperm at a concentration of $2 \times 10^{6}$ cells $/ \mathrm{mL}$ in $50 \mu \mathrm{L}$ of fertilization medium (Fert-TALP). The fertilization medium consisted of FertTALP medium supplemented with $0.2 \mathrm{mM} \mathrm{Na-pyruvate,}$ $25 \mu \mathrm{g} / \mathrm{mL}$ gentamycin, and $0.6 \%$ BSA. When sperm were added to the fertilization drops, $10 \mu \mathrm{g} / \mathrm{mL}$ heparin, $80 \mu \mathrm{M}$ penicillamine, $4 \mu \mathrm{M}$ hypotaurine, and $2 \mu \mathrm{M}$ epinephrine (PHE) were also added. After $22 \mathrm{~h}$ of insemination, cumulus-enclosed oocytes were stripped using gentle pipetting and transferred to CR1-aa medium containing $0.4 \mathrm{mM}$ 
Na-pyruvate, $1 \mathrm{mM}$ glutamine, $0.3 \mathrm{mg} / \mathrm{mL}$ glutathione, 25 $\mu \mathrm{g} / \mathrm{mL}$ gentamycin, and $0.3 \%$ BSA for in vitro culture (IVC). After culture for two days, the cleaved embryos were further cultured in medium containing $50 \mu \mathrm{L}$ of CR1-aa 0.4 $\mathrm{mM}$ Na-pyruvate, $1 \mathrm{mM}$ glutamine, $0.3 \mathrm{mg} / \mathrm{mL}$ glutathione, $25 \mu \mathrm{g} / \mathrm{mL}$ gentamycin, and $10 \%$ FBS for five days at $38.5^{\circ} \mathrm{C}$ in $5 \% \mathrm{CO}_{2}$ in air.

\section{Vitrification and warming procedure}

Cryopreservation was carried out by vitrification with a Cryotop (Kitazato Supply Co, Fujinomiya, Japen) using a slightly modified version of the procedure described by Kim et al., 2020. Briefly, two or three blastocysts were transferred into equilibration solution (ES) consisting of $7.5 \%$ ethylene glycol (EG) and $7.5 \%$ dimethylsulfoxide (DMSO) in PBS supplemented with 20\% FBS at room temperature for $5 \mathrm{~min}$. Next, blastocysts were transferred into vitrification solution (VS) consisting of 15\% EG, 15\% DMSO, and $0.5 \mathrm{M}$ sucrose dissolved in PBS containing $20 \%$ FBS. After $40-45 \mathrm{~s}$, the blastocysts were loaded into a Cryotop and plunged into liquid nitrogen. The process from exposure in VS to plunging into liquid nitrogen was completed within $1 \mathrm{~min}$ at room temperature. Vitrified blastocysts were warmed by immersing the Cryotop directly into warming solution (1.0 $\mathrm{M}$ sucrose dissolved in PBS containing $20 \% \mathrm{FBS}$ ) for $1 \mathrm{~min}$, then were transferred to dilution solution ( $0.5 \mathrm{M}$ sucrose dissolved in PBS containing 20\% FBS) for $3 \mathrm{~min}$, and then to dilution solution (0.25 M sucrose dissolved in PBS containing 20\% FBS) for $5 \mathrm{~min}$ at room temperature. Subsequently, blastocysts were incubated for $5 \mathrm{~min}$ in washing solution (PBS containing 20\% FBS). Survival of vitrified-warmed blastocysts was determined according to re-expansion rates after 24 $\mathrm{h}$ of recovery in culture medium. Mito-TEMPO $(0.1 \mu \mathrm{M})$ was added in ES and VS during vitrification periods. In addition, we used fresh bovine embryos and blastocyst (Non-treated group) from in vitro culture (IVC) as controls for comparison with Mito-TEMPO-treated group.

\section{Assessment of apoptosis in bovine blastocysts}

Apoptotic cells in vitrified-warmed blastocysts were detected using an In Situ Cell Death Detection Kit (Roche Diagnostics GmbH, Mannheim, Germany). Vitrifiedwarmed blastocysts were washed with 0.1 PVA in PBS and then fixed in 3.7\% formaldehyde in PBS for 2 days at $4{ }^{\circ} \mathrm{C}$. Next, blastocysts were permeabilized using $0.5 \%$
Triton X-100 for $30 \mathrm{~min}$ at room temperature. The fixed blastocysts were incubated in terminal deoxyribonucleotidyl transferase-mediated dUTP-digoxigenin nick endlabeling (TUNEL) reaction medium for $1 \mathrm{~h}$ at $38.5^{\circ} \mathrm{C}$ in the dark, washed and mounted on slide glass with mounting solution containing $1.5 \mu \mathrm{g} / \mathrm{mL}$ 4', 6-diamidino-2-phenylindole (DAPI; Vector Laboratories, CA, USA). DAPI-labeled or TUNEL-positive nuclei were subsequently observed under a fluorescence microscope (Olympus, Tokyo, Japan). Nuclei were recorded as positive for TUNEL labeling only when they showed light green fluorescence on the blue fluorescence background of DAPI.

\section{Measurement of ROS levels}

The ROS levels in vitrified-warmed blastocysts were measured using the dichlorodihydrofluorescein diacetate (H2DCFDA; Molecular Probes, Eugene, OR, USA) and Mito-SOX (Molecular Probes) as previously described (Lee et al., 2011). Vitrified-warmed blastocysts in CR1aa (with 10\% FBS) medium were washed three times with $0.1 \%$ polyvinyl alcohol (PVA) in phosphate buffer solution (PBS). Blastocysts were transferred into 1X PBS containing $1 \mu \mathrm{M} \mathrm{H} 2 \mathrm{DCFDA}$ and $1 \mu \mathrm{M}$ Mito-SOX for $30 \mathrm{~min}$ at $38.5^{\circ} \mathrm{C}$ under $5 \% \mathrm{CO}_{2}$ in air. The intensity of H2DCFDA and MitoSOX were measured with an iRiS Digital Cell Imaging System (Logos Biosystems, Gyeonggido, South Korea). The measured fluorescence images were analyzed by Image J software Version 1.38 (National Institutes of Health, Bethesda, MD, USA).

\section{MitoTracker Orange staining}

Vitrified-warmed blastocysts were incubated in 1X PBS with $4 \mu \mathrm{M}$ MitoTracker Orange (Invitrogen, CA, USA) for $30 \mathrm{~min}$ at $38.5^{\circ} \mathrm{C}$. Stained blastocysts were fixed in $3.7 \%$ formaldehyde for overnight at $4{ }^{\circ} \mathrm{C}$ and washed thrice with $0.1 \%$ PVA in PBS. The intensity of MitoTracker images were acquired using LSM 800 confocal microscope (Zeiss, Jena, Germany). The mitochondrial activity was measured by orange fluorescence quantification using the Image J 1.46r software (NIH, USA). All images for analysis were taken using the same intensity and exposure time.

\section{Statistical analysis}

All percentage data obtained in the present study are presented as the mean \pm SD. All data were analyzed using student's $t$-tests and plotted using the GraphPad Prism 5.0 
software package (San Diego, CA, USA). Histogram values of densitometry were measured by ImageJ software (NIH, USA).

\section{RESULTS}

\section{Effect of Mito-TEMPO supplementation during vitrification on the survival rates and apoptosis in frozen-thawed bovine embryos}

To investigate the effects of $0.1 \mu \mathrm{M}$ Mito-TEMPO treatment in a freezing medium, we confirmed frozen-thawed bovine blastocyst developmental and re-expanded rates using microscope (Fig. 1). Representative images of the bovine re-expanded blastocyst following vitrified warming are shown in Fig. 1A. The survival rates of frozen-thawed bovine embryos derived from IVF according to MitoTEMPO treatment are shown in Table 1 . As expected, the re-expanded blastocyst rate and survival rate in MitoTEMPO exposed frozen-thawed blastocysts $(56.8 \pm 8.7 \%$, $\mathrm{n}=59)$ were significantly higher $(p<0.001)$ than the nontreated group $(77.5 \pm 8.9 \%, n=63$, Fig. $1 \mathrm{~B}$ and Table 1$)$. We stained nuclei and apoptotic cells using DAPI/TUNEL assay to evaluate frozen-thawed bovine blastocyst quality based on apoptotic positive cells rate (Fig. $2 \mathrm{~A}$ and Table
2). Total number of nuclei in frozen-thawed bovine blastocysts significantly increased $(p<0.01)$ in Mito-TEMPO exposed group $(\mathrm{n}=5.4 \pm 1.2)$ compared with non-treated group ( $\mathrm{n}=6.7 \pm 3.3$, Fig. $2 \mathrm{~B}$ ), whereas TUNEL-positive cells in the Mito-TEMPO-exposed group $(4.2 \pm 1.4 \%)$ was lower than the non-treated group $(7.1 \pm 3.5 \%, p<0.05$, Fig. 2C). These results showed that Mito-TEMPO supplementation in freezing medium improves survival rates through reduction of apoptosis of frozen-thawed bovine embryos.

\section{Mito-TEMPO protects frozen-thawed bovine blastocyst from cryoinjury caused by mitochondrial- derived superoxide}

To examine the expression of intracellular ROS and $\mathrm{mi}^{-}$ tochondrial superoxide, we stained the vitrified-warmed bovine blastocysts in two groups using DCF-DA and MitoSOX staining. There was an obvious decrease in the intracellular ROS level in embryos following vitrification from freezing medium supplied with Mito-TEMPO compared to the non-treated group ( $p<0.05$, Fig. 3A and 3B). A representative image of the mitochondrial superoxide detection by Mito-SOX staining in the vitrified-warmed blastocysts are shown in Fig. 3A. Decrease in mitochon-
A

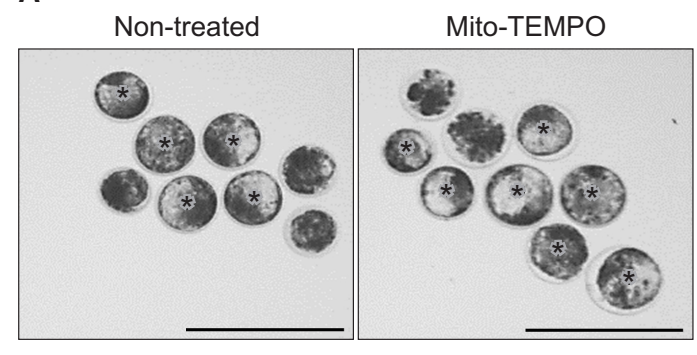

*Blastocyst formation

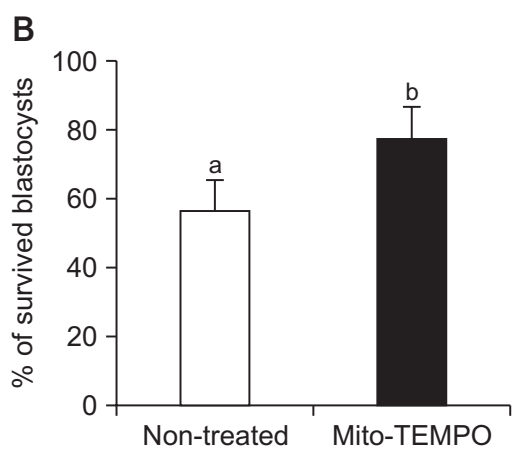

Fig. 1. Effects of Mito-TEMPO on blastocyst survival competence in vitrified-warmed bovine blastocysts. (A) Representative images of survived blastocysts according to the Mito-TEMPO treatment in vitrified-warmed blastocysts. ${ }^{*}$ (asterisks) indicates survived blastocysts after vitrified-warmed process. (B) The percentage of survival rates regard to the Mito-TEMPO exposed in vitrified-warmed bovine blastocysts. Data are expressed as mean \pm SD and were analyzed using a t-test. Different superscript letters a and $b$ denote significant differences at $p<0.05$.

Table 1. Survival rates of vitrified-warmed bovine blastocysts with Mito-TEMPO

\begin{tabular}{lccc}
\hline Mito-TEMPO $(\mu \mathrm{M})$ & No. of blastocysts vitrified & No. of blastocysts thawed & \% of survived blastocysts $(\mathrm{n})$ \\
\hline Non-treated & 62 & 59 & $56.8 \pm 8.7(35)^{\mathrm{a}}$ \\
0.1 & 63 & 63 & $77.5 \pm 8.9(49)^{b}$ \\
\hline
\end{tabular}

Data are expressed as the mean \pm SD, and non-normally distributed data are expressed as the median (interquartile range). Different superscript letters a and $b$ denote significant differences $(p<0.05)$. 
A

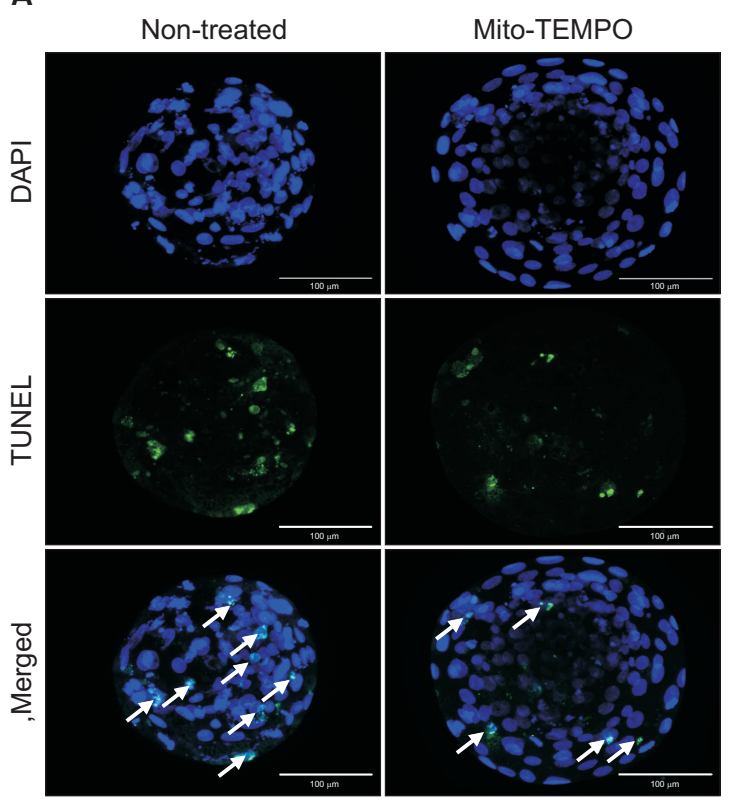

B

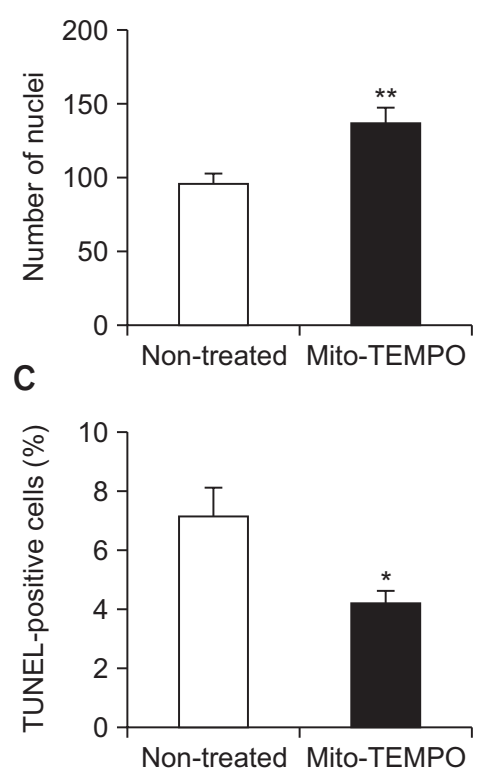

Fig. 2. Changes in cellular apoptosis according to the Mito-TEMPO treatment on vitrified-warmed blastocysts in bovine. (A) Colocalization of DAPI and TUNEL stains at the vitrifiedwarmed blastocyst stage. The blastocysts were stained for nuclei (blue) and TUNEL-positive cells (green). The white arrows indicate a fragmented DNA of nucleus in blastocysts. (B) The graph indicates the number of nucle in vitrified-warmed bovine blastocysts. (C) The percentage of TUNEL-positive cells rates depending on the MitoTEMPO treatment in bovine vitrifiedwarmed blastocysts are shown. Data are expressed as mean \pm SD and were analyzed using a t-test. Differences were considered significant at ${ }^{*} p<$ 0.05 and ${ }^{* *} p<0.01$.
Table 2. TUNEL-positive cells rates of frozen-thawed bovine blastocysts in Mito-TEMPO treatment

\begin{tabular}{lcc}
\hline Mito-TEMPO $(\mu \mathrm{M})$ & $\begin{array}{c}\text { No. of TUNEL- } \\
\text { positive cells }\end{array}$ & $\begin{array}{c}\text { \% of TUNEL- } \\
\text { positive cells }\end{array}$ \\
\hline Non-treated & $6.7 \pm 3.3$ & $7.1 \pm 3.5$ \\
0.1 & $5.4 \pm 1.2$ & $4.2 \pm 1.4^{*}$ \\
\hline
\end{tabular}

Data are expressed as the mean \pm SD, and non-normally distributed data are expressed as the median (interquartile range). Differences were considered significant at ${ }^{*} p<0.05$.

drial superoxide production was clearly observed in MitoTEMPO exposed embryos following cryopreserved-thawing ( $p<0.05$, Fig. 3C and 3D). Based on these results, we demonstrated that Mito-TEMPO decreased the oxidative stress from intracellular ROS and mitochondrial superoxide in vitrified-warmed bovine embryos during cryopreservation process.

\section{Mito-TEMPO improves the mitochondrial activity in re-expanded bovine blastocysts after cryopreservation Mitochondria activity was evaluated by MitoTracker Or- ange staining in frozen-thawed bovine from Mito-TEMPO treated and non-treated group. The overall intensity of MitoTracker Orange fluorescence expression in blasto- cysts were significantly increased in the Mito-TEMPO- supplemented group compared with the non-treated group ( $p<0.05$, Fig. 4A and 4B).}

\section{DISCUSSION}

In current study, we investigated the effects of MitoTEMPO through reduction of mitochondrial specific superoxide production using freezing medium supplemented with $0.1 \mu \mathrm{M}$ Mito-TEMPO for improving survival rate and quality in bovine blastocyst following cryopreservedthawing. Freezing medium with Mito-TEMPO treatment enhances survival and re-expanding rates of blastocysts through reduction of apoptosis, intracellular ROS, and mitochondrial superoxide in bovine embryos following vitrification. These findings suggest the first evidence related to protective effect of Mito-TEMPO in freezing medium for enhancing survival rate of cryopreserved-thawed bovine blastocyst.

To improve the challenges of current bovine reproduction, many studies have been focusing on increasing cryo-survival rates of in vitro produced embryos (IVP) involved in the resumption of development after cryopreservation (Gupta and Lee, 2010; Marsico et al., 2019; Stoecklein et al., 2021). Previous study clearly indicated that thawed blastocyst after cryopreservation shows apoptotic response such as DNA fragmentation compared to in vitro blastocysts (Majidi Gharenaz et al., 2016). In present study, we evaluated the ability of re-expansion and survival in vitrified-warmed bovine embryos and correlated them with the apoptotic status derived from ROS and superoxide production (Fig. 1-3). Increased apoptotic 
A
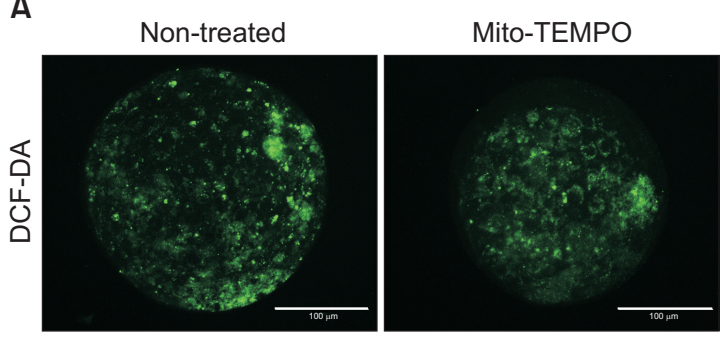

C
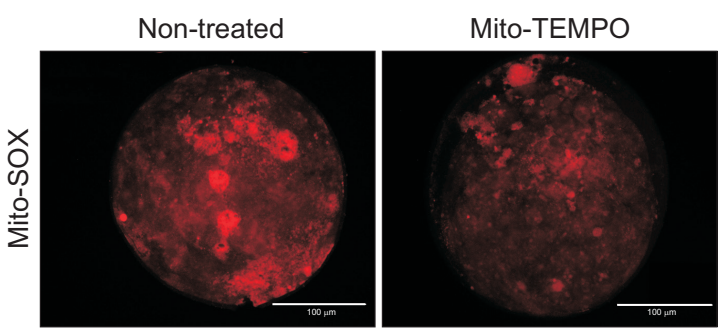

B

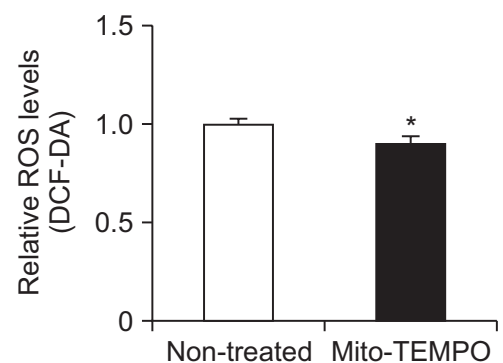

D

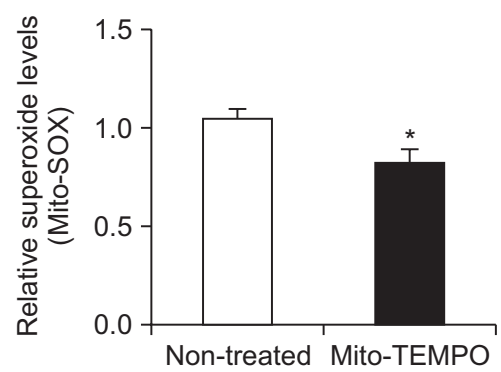

Fig. 3. Comparison of intracellular and mitochondrial ROS expressions in frozen-thawed bovine blastocysts treated Mito-TEMPO. (A) Expression of intracellular ROS (green fluorescence) in vitrified-warmed bovine blastocysts was detected by DCF-DA and analysis using fluorescence microscope. (B) The graph showed relative ROS expression levels on vitrified-warmed blastocyst in bovine. (C) Representative images of mitochondrial superoxide (red fluorescence) in vitrifiedwarmed bovine blastocysts detected by Mito-SOX and analysis using image system. (D) Relative superoxide expression levels of vitrified-warmed bovine blastocysts treated Mito-TEMPO. Data are expressed as mean \pm SD and were analyzed using a t-test. Differences were considered significant at ${ }^{*} p<0.05$
A

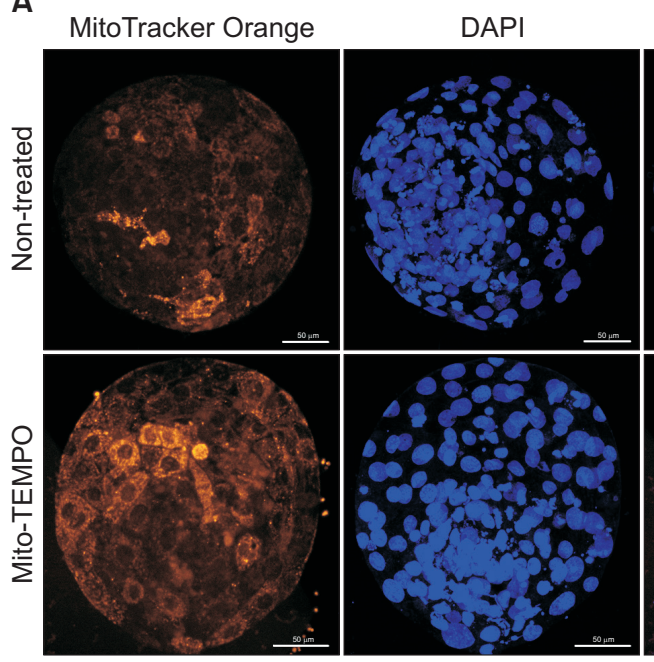

Merged

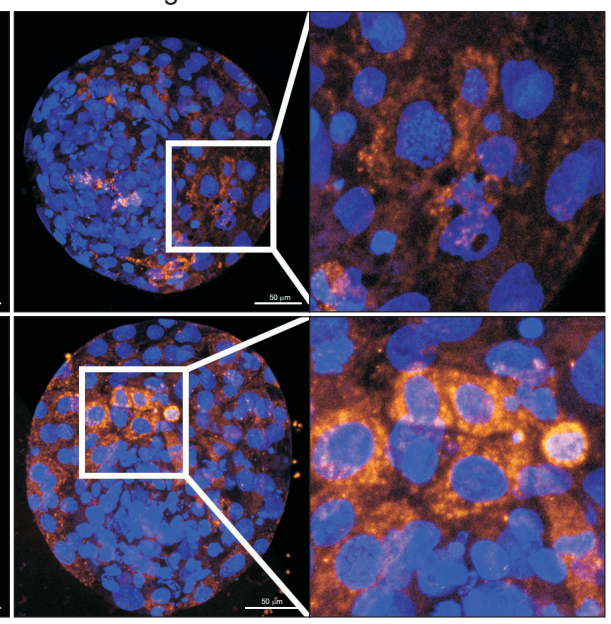

B

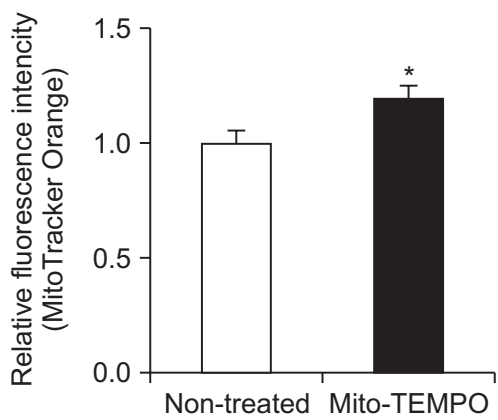

Fig. 4. Confirmation of mitochondrial activity and localization in the frozen-thawed blastocysts with Mito-TEMPO. (A) Representative images of Mitochondria intensity (orange fluorescence) by MitoTracker Orange staining following the Mito-TEMPO treatment in frozen-thawed blastocysts. Mitochondrial expressions and localization were visualized using a fluorescence microscope. (B) The graph indicated relative fluorescence intensity of mitochondria of bovine frozen-thawed blastocyst. Data are expressed as mean \pm SD and were analyzed using a t-test. Differences were considered significant at ${ }^{*} p<0.05$.

cells by vitrification-induced abnormal or excessive ROS generation and DNA damage-induced phosphorylated histone H2A $(\gamma-\mathrm{H} 2 \mathrm{AX})$ accumulation induced disruption of early embryonic developmental competence until expanded blastocysts (Chang et al., 2019). Collectively, low survival rate of vitrified embryos after warming suggests that cryoinjury aggravates embryo quality and developmental capacity through the oxidative stress derived serve
ROS accumulation.

Many antioxidants have been effectively employed as a cryoprotective supplement for bovine embryo or frozenthawed sperm preservation (Al-Mutary, 2021). In previous our study, it is suggested that $0.1 \mu \mathrm{M}$ Mito-TEMPO exposure can improve early embryonic developmental capacity through mitochondrial superoxide production (Yang et al., 2018). However, effects of Mito-TEMPO supplement 
have not yet been assessed for bovine blastocyst during freezing process. In the present study, we confirmed that supplementation with $0.1 \mu \mathrm{M}$ of Mito-TEMPO reduced intracellular ROS and superoxide from mitochondria in frozen-thawed bovine blastocysts.

The MitoTracker Orange staining is commonly been used to measure mitochondrial activity of embryos (Elahi et al., 2017). It was suggested that the cryopreservation process using bovine embryo or blastocyst could cause irreversible damage to cell organelles, including mitochondria which leads to apoptosis (Gualtieri et al., 2021). Damaged mitochondria have been found to accelerate mitochondrial ROS production, oxidative damage, and disruption of mitochondrial bioenergetics based on low mitochondrial activation (Guo et al., 2013). Our findings demonstrate that viability and mitochondrial activity of frozen-thawed embryos with $0.1 \mu \mathrm{M}$ Mito-TEMPO were enhanced when compared to non-treated group (Fig. 4). In addition, analysis of mitochondrial activation also showed that Mito-TEMPO supplementation could significantly increase the survival rate of frozen-thawed bovine blastocyst by regulating mitochondrial ROS production. These results suggest that the regulation of Mito-TEMPO between mitochondrial activation and superoxide production in frozen-thawed bovine embryos could enhance viability and survival capacity during cryopreservation.

\section{CONCLUSION}

In conclusion, our findings showed that improvement of survivability in vitrified bovine embryos through reducing mitochondrial superoxide during vitrification process supplemented with Mito-TEMPO. In the present study, it is speculated that cryopreservation could be responsible for ROS and superoxide production which results in apoptosis and reduction of mitochondrial activity. Therefore, our results suggested the potential of Mito-TEMPO as a supplement for improving frozen-thawed blastocysts survival rates in bovine embryos.

Author Contributions: Conceptualization, J.H.J., H.J.P., and D.B.K.; methodology, J.H.J. and S.G.Y.; investigation, J.H.J. and S.G.Y.; data curation, J.H.J., S.G.Y., and H.J.P.; writing - original draft, J.H.J. and S.G.Y.; writing - review \& editing, S.G.Y., H.J.P., and D.B.K.; supervision, H.J.P. and D.B.K.; project administration, J.H.J., S.G.Y., and H.J.P.; funding acquisition, H.J.P. and D.B.K.

Funding: This research was supported by the Basic Science Research Program through the National Research Foundation of Korea (NRF-2021R1C1C2009469 and NRF2019R1A2C1085199) funded by and the Ministry of Science and ICT, Republic of Korea.

Ethical Approval: Not applicable.

Consent to Participate: Not applicable.

Consent to Publish: Not applicable.

Availability of Data and Materials: Not applicable.

Acknowledgements: None.

Conflicts of Interest: No potential conflict of interest relevant to this article was reported.

\section{REFERENCES}

Al-Mutary MG. 2021. Use of antioxidants to augment semen efficiency during liquid storage and cryopreservation in livestock animals: a review. J. King Saud Univ. Sci. 33:101226.

Asadzadeh N, Abdollahi Z, Esmaeilkhanian S, Masoudi R. 2021. Fertility and flow cytometry evaluations of ram frozen semen in plant-based extender supplemented with MitoTEMPO. Anim. Reprod. Sci. 233:106836.

Babayev E and Seli E. 2015. Oocyte mitochondrial function and reproduction. Curr. Opin. Obstet. Gynecol. 27:175-181.

Chang H, Chen H, Zhang L, Wang Y, Xie X, Zhang Y, Quan F. 2019. Effect of oocyte vitrification on DNA damage in metaphase II oocytes and the resulting preimplantation embryos. Mol. Reprod. Dev. 86:1603-1614.

Choumar A, Tarhuni A, Lettéron P, Reyl-Desmars F, Dauhoo N, Damasse J, Vadrot N, Nahon P, Moreau R, Pessayre D, Mansouri A. 2011. Lipopolysaccharide-induced mitochondrial DNA depletion. Antioxid. Redox Signal. 15:2837-2854.

Dikalova AE, Bikineyeva AT, Budzyn K, Nazarewicz RR, McCann L, Lewis W, Harrison DG, Dikalov SI. 2010. Therapeutic targeting of mitochondrial superoxide in hypertension. Circ. Res. 107:106-116.

Elahi F, Shin H, Lee J, Lee E. 2017. Endoplasmic stress inhibition during oocyte maturation improves preimplantation development of cloned pig embryos. J. Emb. Trans. 32:287295.

Gualtieri R, Kalthur G, Barbato V, Di Nardo M, Adiga SK, Talevi R. 2021. Mitochondrial dysfunction and oxidative stress 
caused by cryopreservation in reproductive cells. Antioxidants (Basel) 10:337.

Guo C, Sun L, Chen X, Zhang D. 2013. Oxidative stress, mitochondrial damage and neurodegenerative diseases. Neural Regen. Res. 8:2003-2014.

Gupta MK and Lee HT. 2010. Cryopreservation of oocytes and embryos by vitrification. Korean J. Reprod. Med. 37:267-291.

Hara T, Kin A, Aoki S, Nakamura S, Shirasuna K, Kuwayama T, Iwata H. 2018. Resveratrol enhances the clearance of mitochondrial damage by vitrification and improves the development of vitrified-warmed bovine embryos. PLoS One 13:e204571.

Huebinger J, Han HM, Hofnagel O, Vetter IR, Bastiaens PI, Grabenbauer M. 2016. Direct measurement of water states in cryopreserved cells reveals tolerance toward ice crystallization. Biophys. J. 110:840-849.

Inaba Y, Miyashita S, Somfai T, Geshi M, Matoba S, Dochi O, Nagai T. 2016. Cryopreservation method affects DNA fragmentation in trophectoderm and the speed of re-expansion in bovine blastocysts. Cryobiology 72:86-92.

Kim JW, Yang SG, Park HJ, Kim JH, Lee DM, Woo SM, Kim HJ, Kim HA, Jeong JH, Lee MJ, Koo DB. 2020. Comparison of Cryotop and ReproCarreir products for cryopreservation of bovine blastocysts through survival rate and blastocysts quality. J. Anim. Reprod. Biotechnol. 35:207-213.

Lee HL, Kim SH, Kim YJ. 2014. Studies on cryotop vitrification method for simple freezing of Hanwoo embryos. J. Emb. Trans. 29:13-19.

Lee KS, Kim EY, Jeon K, Cho SG, Han YJ, Yang BC, Lee SS, Ko MS, Riu KJ, Lee HT, Park SP. 2011. 3,4-Dihydroxyflavone acts as an antioxidant and antiapoptotic agent to support bovine embryo development in vitro. J. Reprod. Dev. 57:127-134.

Lee S, Park HW, Cheong HT, Park CK, Yang BK. 2016. Effects of turine and vitamin $\mathrm{E}$ on sperm viability, membrane integrity and mitochondrial activity damaged by bromopropane in fresh boar semen. J. Emb. Trans. 31:13-17.

Len JS, Koh WSD, Tan SX. 2019. The roles of reactive oxygen species and antioxidants in cryopreservation. Biosci. Rep. 39:BSR20191601.

Lu X, Zhang Y, Bai H, Liu J, Li J, Wu B. 2018. Mitochondriatargeted antioxidant MitoTEMPO improves the post-thaw sperm quality. Cryobiology 80:26-29.

Majidi Gharenaz N, Movahedin M, Mazaheri Z, Pour Beiranvand S. 2016. Alternation of apoptotic and implanting genes expression of mouse embryos after re-vitrification. Int. J. Reprod. Biomed. 14:511-518.

Marsico TV, de Camargo J, Valente RS, Sudano MJ. 2019. Embryo competence and cryosurvival: molecular and cellular features. Anim. Reprod. 16:423-439.

Masoudi R, Asadzadeh N, Sharafi M. 2021. Effects of freezing extender supplementation with mitochondria-targeted antioxidant Mito-TEMPO on frozen-thawed rooster semen quality and reproductive performance. Anim. Reprod. Sci. 225:106671.

May-Panloup P, Boguenet M, Hachem HE, Bouet PE, Reynier P. 2021. Embryo and its mitochondria. Antioxidants (Basel) 10:139.

Nohales-Córcoles M, Sevillano-Almerich G, Di Emidio G, Tatone C, Cobo AC, Dumollard R, De Los Santos Molina MJ. 2016. Impact of vitrification on the mitochondrial activity and redox homeostasis of human oocyte. Hum. Reprod. 31:1850-1858.

Ott M, Gogvadze V, Orrenius S, Zhivotovsky B. 2007. Mitochondria, oxidative stress and cell death. Apoptosis 12:913-922.

Park HJ, Song BS, Kim JW, Yang SG, Kim SU, Koo DB. 2020. Exposure of triclosan in porcine oocyte leads to superoxide production and mitochondrial-mediated apoptosis during in vitro maturation. Int. J. Mol. Sci. 21:3050.

Shadel GS and Horvath TL. 2015. Mitochondrial ROS signaling in organismal homeostasis. Cell 163:560-569.

Stoecklein KS, Ortega MS, Spate LD, Murphy CN, Prather RS. 2021. Improved cryopreservation of in vitro produced bovine embryos using FGF2, LIF, and IGF1. PLoS One 16:e0243727.

van Hameren G, Campbell G, Deck M, Berthelot J, Gautier B, Quintana P, Chrast R, Tricaud N. 2019. In vivo real-time dynamics of ATP and ROS production in axonal mitochondria show decoupling in mouse models of peripheral neuropathies. Acta Neuropathol. Commun. 7:86.

Xia M, Zhang Y, Jin K, Lu Z, Zeng Z, Xiong W. 2019. Communication between mitochondria and other organelles: a brand-new perspective on mitochondria in cancer. Cell Biosci. 9:27.

Yang SG, Park HJ, Kim JW, Jung JM, Kim MJ, Jegal HG, Kim IS, Kang MJ, Wee G, Yang HY, Lee YH, Seo JH, Kim SU, Koo DB. 2018. Mito-TEMPO improves development competence by reducing superoxide in preimplantation porcine embryos. Sci. Rep. 8:10130.

Yang SG, Park HJ, Lee SM, Kim JW, Kim MJ, Kim IS, Jegal HG, Koo DB. 2019. Reduction of mitochondrial derived superoxide by Mito-TEMPO improves porcine oocyte maturation in vitro. J. Anim. Reprod. Biotechnol. 34:10-19.

Yousefian I, Zare-Shahneh A, Goodarzi A, Baghshahi H, Fouladi-Nashta AA. 2021. The effect of Tempo and MitoTEMPO on oocyte maturation and subsequent embryo development in bovine model. Theriogenology 176:128-136. 